\title{
Editorial: Role of Inner Ear in Self and Environment Perception
}

\author{
Christophe Lopez ${ }^{1 *}$, Michel Toupet ${ }^{2,3}$, Christian van Nechel ${ }^{3,4}$ and Alexis Bozorg Grayeli ${ }^{5,6 *}$ \\ ${ }^{1}$ Aix-Marseille University, CNRS, LNSC, Marseille, France, ${ }^{2}$ Centre d'Explorations Fonctionnelles Otoneurologiques, Paris, \\ France, ${ }^{3}$ Institut de Recherche Oto-Neurologique (IRON), Paris, France, ${ }^{4}$ Clinique des Vertiges, Brussels, Belgium, \\ ${ }^{5}$ Otolaryngology Department, Dijon University Hospital, Université Bourgogne-Franche Comté, Dijon, France, ${ }^{6}$ Electronic, \\ Image and Computer Research Laboratory, Le2i, Dijon, France
}

Keywords: self-motion, vestibular system, vestibular rehabilitation, postural control, visual vertical, depersonalization and derealization, dizziness and vertigo, nystagmus

\section{Editorial on the Research Topic}

\section{Role of Inner Ear in Self and Environment Perception}

Otoneurology and vestibular neuroscience recently advanced with a better understanding of the vestibular contributions to perceptual and cognitive functions, reaching far beyond balance, and eye-movement control. Pioneering clinical observations established connections between dizziness and altered sense of self, distortions of the body schema, and symptoms resembling depersonalization and derealization $(1,2)$. However, studies in large samples of patients with dizziness have only recently validated the assumption that the vestibular system is the main contributor to the bodily self $(3,4)$. Recent epidemiological studies have also linked vestibular disorders to cognitive deficits. For example, a survey in over 20,000 adults established that individuals reporting vestibular vertigo had an eight-fold increase in the odds of reporting impaired memory and attention, limiting their activities (5). Another survey conducted in adults over 60 years revealed that vestibular impairment partially mediated the association between age and cognitive impairment. It was estimated that vestibular impairment mediates $14.3 \%$ of the effects of age on cognition and that it accelerates cognitive decline by 5 years in a visuo-spatial test (6). These studies raise the necessity to investigate more carefully the effects of vestibular impairment in dementia and several psychiatric disorders $(7,8)$.

Research in this area benefited from a better delineation of the human vestibular cortex. Functional MRI (fMRI) studies and meta-analyses of neuroimaging data have revealed that the cortical vestibular network is centered on the operculo-insular/retroinsular cortex (9) and that vestibular inputs also project to the temporo-parietal junction, cingulate cortex, somatosensory cortex, posterior parietal cortex, hippocampus, and frontal eye fields (10). These widespread vestibular projections to the brain were recently confirmed in whole-brain functional mapping in rodents using fMRI, local field potentials, and optogenetics $(11,12)$. We note that recent descriptions of functional connectivity, metabolic, and morphological brain alterations in peripheral vestibular disorders or chronic subjective dizziness [e.g., (13-15)] offer the possibility to evaluate, in a non-invasive manner, how various vestibular rehabilitation methods and drugs can improve brain plasticity. Finally, recent fMRI studies revealed the influence of cognition, emotion, and personality traits (such as neuroticism and introversion) on vestibular information processing [e.g., $(16,17)]$. These observations suggest an expansion of the vestibular brain network into dimensions of emotion processing, mental health, and social cognition.

This Research Topic collection includes 17 articles combining contributions from authors with a large range of expertise in medicine and basic science, including neurology, otorhinolaryngology, neurophysiology, physiotherapy, neuropsychology, cognitive neuroscience, and bioengineering. 
This initiative brought together authors affiliated to institutions from six continents and 17 countries (Belgium, Bulgaria, Canada, France, Germany, Iran, Italy, Ivory Coast, Mexico, Netherlands, New Zealand, Russia, Spain, Switzerland, Taiwan, the United Kingdom, and the United States), showing the worldwide interest in advancing research in otoneurology, vestibular physiology, and vestibular cognition.

Altogether, contributions from this Research Topic highlight recent discoveries in otoneurology regarding (1) upright perception; (2) self-motion perception and balance control; (3) self and own-body cognition; and (4) vestibular physiopathology, testing, and rehabilitation.

\section{UPRIGHT PERCEPTION: FROM NEUROIMAGING TO CLINICAL ASSESSMENT}

An important function of the vestibular system is the perception of the visual vertical (VV), which contributes not only to ownbody orientation and postural control but also to the judgment of orientation and even stability of objects in the environment. Psychophysical and clinical studies showed that vestibular signals are integrated with visual, tactile, proprioceptive, and interoceptive signals for an accurate VV perception, probably following Bayesian rules (18). In line with a developmental and multisensory perspective, Cuturi and Gori document visual, haptic, and visuo-haptic vertical perception in children and young adults tilted $90^{\circ}$ to their side. Bayesian modeling reveals a lack of multisensory integration in children for the bimodal task.

Dieterich and Brandt offer a comprehensive overview of the pathways underpinning $\mathrm{VV}$ perception from the inner ear to the cerebral cortex, describing the consequences of lesions of various structures along these pathways. Using fMRI in healthy adults, Saj et al. show that VV perception involves the temporo-occipital and parieto-occipital networks (including the cuneus, lingual gyrus, and precuneus), together with areas in the cerebellum and brainstem, in accordance with observations in neurological patients $(19,20)$. Similar networks have been involved in navigation, balance control, and body representations, suggesting overlaps between these functions and VV perception.

Although VV perception is a widely used test of peripheral vestibular functions, it is not commonly used in neurological conditions such as vestibular migraine. Winnick et al. found that patients with vestibular migraine, but normal vestibular function, show abnormal VV when their head is tilted to the right (as if they were further tilted), whereas their VV is normal with their head upright or tilted to the left. This is corroborated by symptoms (tilting, pulling, and rotation) reported by the same patients mainly to their right side. These observations indicate abnormal multisensory integration for VV perception in vestibular migraine, consistent with abnormal self-motion perception recently measured in vestibular migraine (21)

\section{SELF-MOTION PERCEPTION AND BALANCE CONTROL: FROM PSYCHOPHYSICS TO CLINICAL ASSESSMENT}

Self-motion perception also involves several motion sensors and multisensory brain areas. Its exploration is crucial to understand self-environment interactions (22). Britton and Arshad thoroughly review human and animal studies of the relations between sensory signals and processing centers, as well as their impact on cognitive functions, such as navigation, spatial awareness, and emotions. Although many connections are to be explored, the authors present the system from an interesting perspective of integrative physiology. Kolev analyzed interindividual differences in the perception of self-motion vs. environment-motion perception after a rotatory chair stimulus. The author reports a significant effect of insinuation and sex on motion perception, opening insights into the mechanisms of motion sickness.

Two studies describe sensorimotor adaptations underpinning self-motion perception and balance control in patients with a bilateral vestibular failure (BVF). Lacour et al. show that BVF patients improve balance during fixation of a target, as well as during pursuit of a target with slow eye movements and saccades. They propose that BVF patients may use more efficiently proprioception from extraocular muscles or efference copy from the eye motor command than healthy controls. Guigou et al. explore the effect of a rotating sound on balance. A rotating sound destabilizes patients with BVF and with bilateral cochlear implants, indicating that hearing can also be used for postural control in patients with sensory deficits.

Magnetic vestibular stimulation in an MRI scanner can also evoke sensations of self-motion and horizontal nystagmus (23). Ward et al. show that visual information and continuous head rotations in the magnetic field do not influence the set-point adaptation (i.e., mechanisms attempting to inhibit unwanted nystagmus) of the nystagmus. This is in contrast with the wellknown effect of vision on adaptation of the vestibulo-ocular reflex (VOR).

\section{SELF AND OWN-BODY COGNITION}

Three original research articles highlight the increasingly recognized role of the vestibular system in the sense of self and in own-body cognition. In a large sample of 319 patients with chronic dizziness, Toupet et al. show increased symptoms of depersonalization-derealization with increased levels of anxiety and depression. In addition, they show that depersonalizationderealization is associated with higher visual and vestibular hypersensitivity, migraine, and motion sickness. Aranda-Moreno et al. report that caloric vestibular stimulation and unilateral centrifugation both decreased phantom limb pain and symptoms of depersonalization-derealization reported by amputees. In an original study conducted in healthy participants, Thür et al. adapt the full-body illusion, a visuo-tactile illusion of self-identification with a virtual character (24), to include sensory conflicts between 
gravitational and visual cues. Their results suggest a mutual interaction of graviceptive and other sensory signals and the individual's weighting style in defining the sense of self.

\section{ADVANCES IN VESTIBULAR PHYSIOPATHOLOGY, TESTING, AND REHABILITATION}

Vestibular dysfunctions have been associated with anxiety and hyperactivity (25), premature cognitive decline, or Alzheimer's disease (26). Other neurological disorders may also be partly related to vestibular disorders. Here, Smith summarizes recent research in humans and animals, establishing connections between the vestibular system and Parkinson's disease.

Standard otoneurological evaluation of the peripheral vestibular system now includes the video head impulse test, which is useful to detect vestibular hypofunction by testing the VOR during high-velocity head rotations (27). In a study conducted in BVF patients, Van Nechel et al. analyze the factors influencing the catch-up saccades (visible target vs. in darkness with imaginary target), which are generated to compensate deficient VOR. The authors propose that visual signals are the main trigger and parametric determinant of the catch-up saccades and that a target is necessary in most cases to generate catch-up saccades.

\section{REFERENCES}

1. Bonnier P. Le Vertige. Paris: Rueff (1893).

2. Schilder P. The Image and Appearance of the Human Body. New York, NY: International Universities Press (1935).

3. Jauregui-Renaud K. Vestibular function and depersonalization/derealization symptoms. Multisens Res. (2015) 28:637-51. doi: 10.1163/22134808-00002480

4. Lopez C, Elzière M. Out-of-body experience in vestibular disorders - a prospective study of 210 patients with dizziness. Cortex. (2018) 104:193206. doi: 10.1016/j.cortex.2017.05.026

5. Bigelow RT, Semenov YR, du Lac S, Hoffman HJ, Agrawal Y. Vestibular vertigo and comorbid cognitive and psychiatric impairment: the 2008 National Health Interview Survey. J Neurol Neurosurg Psychiatr. (2016) 87:367-72. doi: 10.1136/jnnp-2015-310319

6. Semenov YR, Bigelow RT, Xue QL, du Lac S, Agrawal Y. Association between vestibular and cognitive function in U.S. adults: data From the National Health and Nutrition Examination Survey. J Gerontol A Biol Sci Med Sci. (2016) 71:243-50. doi: 10.1093/gerona/glv069

7. Gurvich C, Maller JJ, Lithgow B, Haghgooie S, Kulkarni J. Vestibular insights into cognition and psychiatry. Brain Res. (2013) 1537:24459. doi: 10.1016/j.brainres.2013.08.058

8. Previc FH. Vestibular loss as a contributor to Alzheimer's disease. Med Hypotheses. (2013) 80:360-7. doi: 10.1016/j.mehy.2012.12.023

9. Frank SM, Greenlee MW. The parieto-insular vestibular cortex in humans: more than a single area? J Neurophysiol. (2018) 120:143850. doi: 10.1152/jn.00907.2017

10. Lopez C, Blanke O. The thalamocortical vestibular system in animals and humans. Brain Res Rev. (2011) 67:11946. doi: 10.1016/j.brainresrev.2010.12.002

11. Rancz EA, Moya J, Drawitsch F, Brichta AM, Canals S, Margrie TW. Widespread vestibular activation of the rodent cortex. J Neurosci. (2015) 35:5926-34. doi: 10.1523/JNEUROSCI.1869-14.2015
In addition to testing reflexive eye movements, Dupuits et al. propose that vestibular evaluation can benefit from systematic measure of vestibular perception thresholds using whole-body motion platforms, as done in standard psychophysics experiments in healthy participants (28). They measured vestibular perceptual thresholds using a hydraulic platform in the dark delivering six translations and six rotations/tilts.

In a perspective of vestibular rehabilitation, Sadeghi et al. show that unidirectional whole-body rotations toward the side of the vestibular deficit decreased VOR asymmetry even $10 \mathrm{~min}$ after one rehabilitation session. In a long-term study, VOR asymmetry decreased to reach normal values during the first two sessions in most patients. Finally, Idoux et al. developed a model to evoke motion sickness in mice and tested how scopolamine (a muscarinic antagonist) can prevent motion sickness at the behavioral and cellular levels. They report that both motion sickness and scopolamine decrease VOR efficacy, which might be a protective mechanism to prevent later occurrences of motion sickness. The authors set the basis for studies of motion sickness in rodents and offer translational perspectives for improving treatment of motion sickness in humans.

\section{AUTHOR CONTRIBUTIONS}

$\mathrm{CL}$ and $\mathrm{AB}$ wrote the manuscript. $\mathrm{CL}, \mathrm{AB}, \mathrm{MT}$, and $\mathrm{CN}$ revised the manuscript.

12. Leong ATL, Gu Y, Chan Y-S, Zheng H, Dong CM, Chan RW, et al. Optogenetic fMRI interrogation of brain-wide central vestibular pathways. Proc Natl Acad Sci USA. (2019) 116:10122-9. doi: 10.1073/pnas.1812453116

13. Becker-Bense S, Dieterich M, Buchholz H-G, Bartenstein P, Schreckenberger $\mathrm{M}$, Brandt T. The differential effects of acute right- vs. left-sided vestibular failure on brain metabolism. Brain Struct Funct. (2014) 219:135567. doi: 10.1007/s00429-013-0573-z

14. Indovina I, Riccelli R, Chiarella G, Petrolo C, Augimeri A, Giofrè L, et al. Role of the insula and vestibular system in patients with chronic subjective dizziness: an fMRI study using sound-evoked vestibular stimulation. Front Behav Neurosci. (2015) 9:334. doi: 10.3389/fnbeh.2015.00334

15. Riccelli R, Passamonti L, Toschi N, Nigro S, Chiarella G, Petrolo C, et al. Altered insular and occipital responses to simulated vertical self-motion in patients with persistent postural-perceptual dizziness. Front Neurol. (2017) 8:529. doi: 10.3389/fneur.2017.00529

16. Indovina I, Riccelli R, Staab JP, Lacquaniti F, Passamonti L. Personality traits modulate subcortical and cortical vestibular and anxiety responses to sound-evoked otolithic receptor stimulation. J Psychosom Res. (2014) 77:391400. doi: 10.1016/j.jpsychores.2014.09.005

17. Riccelli R, Indovina I, Staab JP, Nigro S, Augimeri A, Lacquaniti F, et al. Neuroticism modulates brain visuo-vestibular and anxiety systems during a virtual rollercoaster task. Hum Brain Mapp. (2017) 38:71526. doi: 10.1002/hbm.23411

18. Angelaki DE, Klier EM, Snyder LH. A vestibular sensation: probabilistic approaches to spatial perception. Neuron. (2009) 64:448-61. doi: 10.1016/j.neuron.2009.11.010

19. Baier B, Bense S, Dieterich M. Are signs of ocular tilt reaction in patients with cerebellar lesions mediated by the dentate nucleus? Brain. (2008) 131:144554. doi: 10.1093/brain/awn086

20. Yang TH, Oh SY, Kwak K, Lee JM, Shin BS, Jeong SK. Topology of brainstem lesions associated with subjective visual vertical tilt. Neurology. (2014) 82:1968-75. doi: 10.1212/WNL.0000000000000480 
21. King S, Priesol AJ, Davidi SE, Merfeld DM, Ehtemam F, Lewis RF. Self-motion perception is sensitized in vestibular migraine: pathophysiologic and clinical implications. Sci Rep. (2019) 9:14323. doi: 10.1038/s41598-019-50803-y

22. Cullen KE. Vestibular processing during natural self-motion: implications for perception and action. Nat Rev Neurosci. (2019) 20:346-63. doi: 10.1038/s41583-019-0153-1

23. Roberts DC, Marcelli V, Gillen JS, Carey JP, Della Santina CC, Zee DS. MRI magnetic field stimulates rotational sensors of the brain. Curr Biol. (2011) 21:1635-40. doi: 10.1016/j.cub.2011.08.029

24. Lenggenhager $B$, Tadi $T$, Metzinger $T$, Blanke $O$. Video ergo sum: manipulating bodily self-consciousness. Science. (2007) 317:1096-9. doi: 10.1126/science.1143439

25. Antoine MW, Vijayakumar S, McKeehan N, Jones SM, Hébert JM. The severity of vestibular dysfunction in deafness as a determinant of comorbid hyperactivity or anxiety. J Neurosci. (2017) 37:5144-54. doi: 10.1523/JNEUROSCI.3545-16.2017

26. Harun A, Oh ES, Bigelow RT, Studenski S, Agrawal Y. Vestibular impairment in dementia. Otol Neurotol. (2016) 37:1137-42. doi: 10.1097/MAO.0000000000001157
27. McGarvie LA, MacDougall HG, Halmagyi GM, Burgess AM, Weber KP, Curthoys IS. The video head impulse test (vHIT) of semicircular canal function - age-dependent normative values of VOR gain in healthy subjects. Front Neurol. (2015) 6:154. doi: 10.3389/fneur.2015.00154

28. Grabherr L, Nicoucar K, Mast FW, Merfeld DM. Vestibular thresholds for yaw rotation about an earth-vertical axis as a function of frequency. Exp Brain Res. (2008) 186:677-81. doi: 10.1007/s00221-008-1350-8

Conflict of Interest: The authors declare that the research was conducted in the absence of any commercial or financial relationships that could be construed as a potential conflict of interest.

Copyright (c) 2020 Lopez, Toupet, van Nechel and Bozorg Grayeli. This is an openaccess article distributed under the terms of the Creative Commons Attribution License (CC BY). The use, distribution or reproduction in other forums is permitted, provided the original author(s) and the copyright owner(s) are credited and that the original publication in this journal is cited, in accordance with accepted academic practice. No use, distribution or reproduction is permitted which does not comply with these terms. 